\title{
Percepción de Discriminación y Obesidad: el Rol de la Afectividad Negativa y la Calidad de la Dieta
}

\section{Perceived Discrimination and Obesity: The Role of Negative Affectivity and Diet Quality}

\author{
Daniela Gómez-Pérez, Belén Salinas-Rehbein, Catalina Becerra-Muñoz y Manuel S. Ortiz \\ Departamento de Psicología, Universidad de La Frontera
}

\begin{abstract}
Diversos factores se asocian con la etiología de la obesidad, dentro de los cuales figuran factores genéticos, psicológicos y conductuales. El objetivo del estudio fue determinar si la discriminación percibida estuvo asociada directamente con obesidad e indirectamente, vía afectividad negativa y calidad de la dieta. Se realizó un muestreo no probabilístico por conveniencia de 420 funcionarios de una universidad regional estatal del sur de Chile (248 mujeres, promedio edad 44 años, $D E=8,79)$, quienes respondieron la escala de percepción de discriminación, estrés psicológico percibido, el inventario de ansiedad rasgo-estado y la escala de depresión CES-D, además de autorreportar la calidad de su dieta. El análisis de ecuaciones estructurales reveló que la discriminación no se relacionó directamente con la obesidad (medida con el perímetro de cintura), pero sí con la afectividad negativa, lo que se vinculó con calidad de la dieta y esta con el perímetro de cintura. Existe una secuencia de mediación de la percepción de discriminación en el perímetro de cintura, vía afectividad negativa y calidad de la dieta que, aunque pequeña en magnitud, es estadísticamente significativa. Se destacan los efectos negativos de la discriminación en salud y se identifica un mecanismo subyacente a esta relación.
\end{abstract}

Palabras clave: percepción de discriminación, obesidad, afectividad negativa, calidad de la dieta

\begin{abstract}
Several risk factors have been linked to the etiology of obesity, including genetic, psychological, and behavioral factors. The aim of the study was to determine whether perceived discrimination was directly associated with obesity, and indirectly via negative affectivity and diet quality. Four hundred and twenty participants from a regional state university in southern Chile were enrolled in the study, using a convenience non-probabilistic sampling procedure $(248$ women, average age 44 years, $S D=8.79)$. Participants completed measures of perceived discrimination, perceived psychological stress, state-trait anxiety, and depressive symptoms (CES-D) and selfreported their diet quality. Structural equation modeling revealed that perceived discrimination was not directly related to obesity (measured using waist size circumference, WSC), however, it was found to be associated with negative affectivity. The latter factor was associated with diet quality, which was itself directly related to WSC. There is a sequenced mediated effect of perceived discrimination on WSC via negative affectivity and diet quality. Though small, this effect is statistically significant. The negative effects of perceived discrimination on health are highlighted and an underlying mechanism linking these variables is identified.
\end{abstract}

Keywords: perceived discrimination, obesity, negative affectivity, diet quality

La obesidad es una enfermedad crónica que constituye la quinta causa de muerte a nivel mundial (Swinburn et al., 2011). Entre 1980 y 2013, su prevalencia se ha incrementado un $28 \%$ en adultos y un $47 \%$ en niños, lo que significa que en las últimas tres décadas las cifras han aumentado de 857 a 2100 millones de personas afectadas por esta condición (Smith \& Smith, 2016). Chile es uno de los países de América Latina con el mayor índice de sobrepeso y obesidad, alcanzando un 63\% de prevalencia en población adulta (Organización de las Naciones Unidas para la Alimentación y la Agricultura \& Organización Panamericana

Daniela Gómez-Pérez (D) https://orcid.org/0000-0002-4407-0567

Belén Salinas-Rehbein (iD https://orcid.org/0000-0001-7604-3101

Catalina Becerra-Muñoz (iD) https://orcid.org/0000-0002-9161-6550

Manuel S. Ortiz iD https://orcid.org/0000-0002-7749-0699

Este estudio fue financiado parcialmente por el proyecto FONDECYT de Iniciación 11140454, FONDECYT Regular 1180463 y el Fondo de Innovación para la Competitividad del Ministerio de Economía, Fomento y Turismo, a través de la Iniciativa Científica Milenio, Proyecto IS130005.

La correspondencia relativa a este artículo debe ser dirigida a Manuel S. Ortiz, Departamento de Psicología, Universidad de La Frontera, Avenida Francisco Salazar 01145, Temuco, Región de la Araucanía, Chile. Email: manuel.ortiz@ufrontera.cl 
de la Salud, 2017). Dicha condición comprende un factor de riesgo para el desarrollo de múltiples patologías, entre las que se destacan la hipertensión arterial, diabetes mellitus tipo 2, enfermedad cardiovascular y el síndrome metabólico (Jakobsen et al., 2018).

Diversos factores se asocian con la etiología de la obesidad, dentro de los cuales figuran factores genéticos, fisiológicos, ambientales, psicológicos y conductuales (Wright \& Aronne, 2012). De las variables psicológicas, la percepción de discriminación en población general es una variable que ha sido estudiada en relación con la obesidad o algunos de sus marcadores objetivos, tanto en población normopeso como con sobrepeso u obesidad. Por ejemplo, en un estudio longitudinal de nueve años se reportó que mujeres y hombres adultos que refirieron percibir diariamente eventos de discriminación, tales como ser tratados con menos respeto que otras personas o recibir un mal servicio (es decir, discriminación debida a diversas causas), aumentaron significativamente su perímetro de cintura (Hunte, 2011). En la misma línea, otro estudio demostró que la discriminación percibida se relaciona con un alto índice de masa corporal y un mayor perímetro de cintura en población afroamericana (Stepanikova et al., 2017) y en forma similar se ha demostrado que estas experiencias se han asociado directamente con obesidad abdominal, tanto en mujeres caucásicas como afroamericanas (Lewis et al., 2011). En consideración de estos antecedentes, se hipotetizó la existencia de un vínculo directo entre percepción de discriminación general y obesidad (ver Figura 1, relación A en el modelo propuesto).

Si bien la evidencia que vincula la percepción de discriminación con obesidad es clara, los mecanismos por medio de los cuales estas variables pueden asociarse han sido menos estudiados. En línea con esto, este estudio viene a responder a este vacío, proponiendo una secuencia de mediación que incluye como mediadores al estrés psicológico, síntomas depresivos y ansiedad, junto con la calidad de la dieta.

Figura 1

Modelo de Relaciones Propuesto

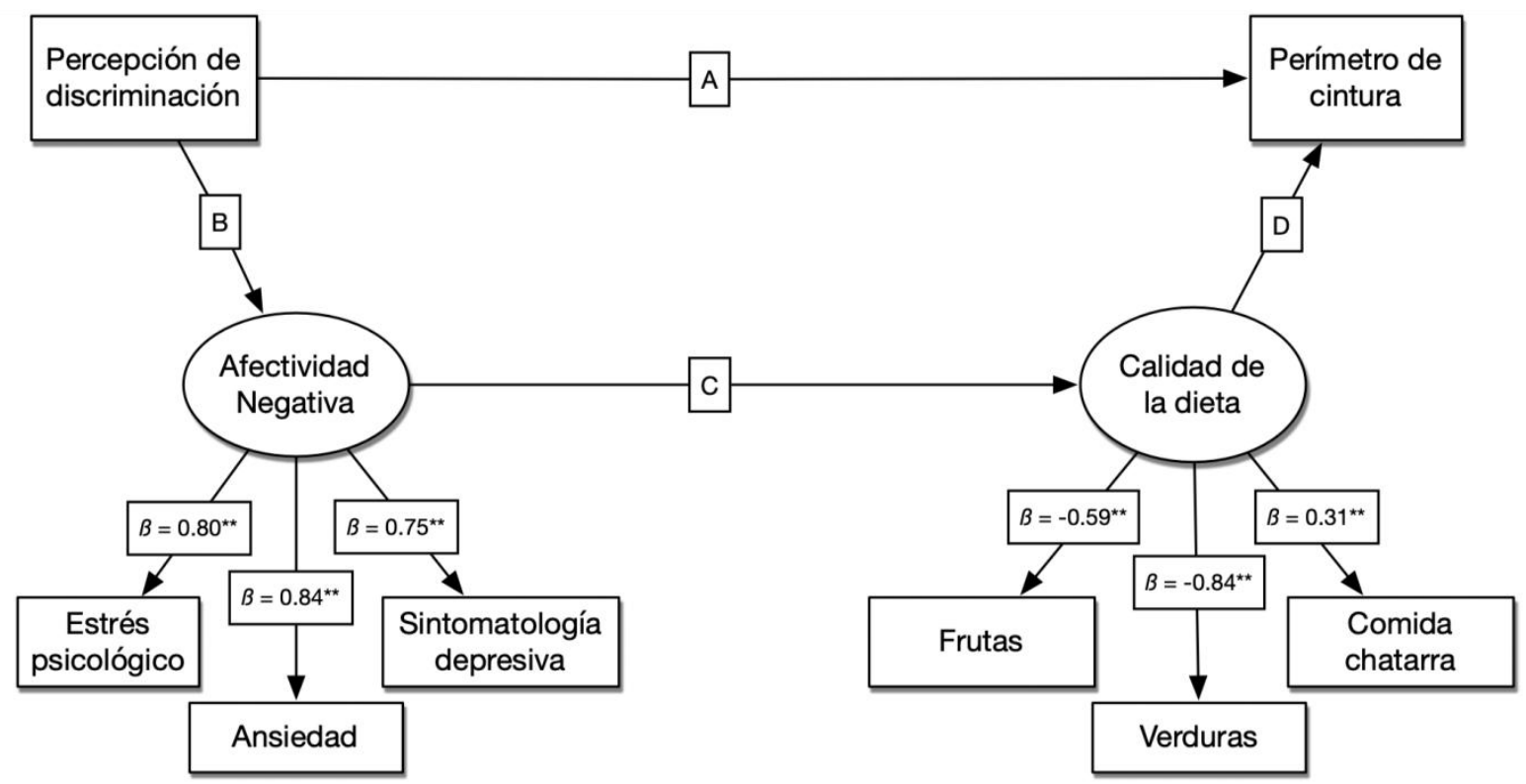




\section{Discriminación Percibida y Afectividad Negativa}

En efecto, se ha reportado que la discriminación percibida puede asociarse con desenlaces en salud, a través de las respuestas afectivas y conductuales motivadas por tales experiencias (Sutin \& Terracciano, 2013). De tal forma, la experiencia subjetiva de ser discriminado ha sido identificada como uno de los factores psicológicos más estresantes y que, simultáneamente, puede influir directa o indirectamente en la salud física de las personas, principalmente porque esta situación es incontrolable, vale decir, no depende de las acciones, sino más bien de la apariencia personal y, además, amenaza la evaluación social que se hace de un individuo (Zeiders et al., 2012). De esta forma, la percepción de discriminación se asocia con resultados negativos en salud mental, lo cual ha sido demostrado en meta-análisis y revisiones sistemáticas. Por ejemplo, en el meta-análisis de Pascoe y Richman (2009), que analiza la asociación entre percepción de discriminación y salud mental en 110 estudios, se reporta una correlación inversa entre ambas variables $(r=-0,16,95 \% \mathrm{CI}=-0,20$ a $-0,12)$, en particular, síntomas depresivos y estrés. Asimismo, una revisión sistemática (Paradies, 2006) demostró una asociación directa entre la experiencia de racismo autorreportado e indicadores negativos de salud mental, tales como síntomas depresivos, estrés psicológico y ansiedad, en $72 \%$ de los 138 estudios incluidos. A esto se suma que la percepción de discriminación ha sido asociada con ansiedad y depresión, tanto en población general (Schmitt et al., 2014) como en personas con un índice de masa corporal mayor a 25 (Hatzenbuehler et al., 2009). En consecuencia, es posible hipotetizar que personas que experimentan mayor percepción de discriminación presentan mayor afectividad negativa, caracterizada por mayor percepción de estrés psicológico, ansiedad y sintomatología depresiva (ver Figura 1, relación B), variables que, por lo tanto, se incluyeron en el modelo propuesto. Esta decisión se basa en la alta correlación que existe entre estas dimensiones, las cuales, además, son frecuentemente estudiadas en conjunto en estudios de similares características.

\section{Afectividad Negativa y la Calidad de la Dieta}

La percepción de estrés psicológico, la sintomatología depresiva y la ansiedad son variables que se han vinculado a múltiples comportamientos en salud, entre los cuales destaca la preferencia por alimentos no saludables (Finch \& Tomiyama, 2015; Gibson-Smith et al., 2018; Joseph et al., 2018). En relación al estrés psicológico, se ha demostrado en forma experimental que ante la generación de alto estrés psicológico, los individuos prefieren alimentos ricos en contenido graso y calórico, por sobre comidas saludables y bajas en grasa (Tomiyama et al., 2015). Adicionalmente, la mayoría de las personas refiere que consume alimentos dulces cuando se siente estresada (Araiza \& Lobel, 2018), resultado que es confirmado en un estudio de 5077 latinos que viven en Estados Unidos, donde se demostró que mayor presencia de estresores crónicos y episódicos se asoció con mayor ingesta de calorías y un patrón de alimentación menos saludable (Isasi et al., 2015).

En relación a la sintomatología depresiva, se ha encontrado que altos niveles de esta variable se asocian al consumo de comida chatarra (Finch \& Tomiyama, 2015) y que, en particular, son las mujeres quienes ingieren la mayor cantidad de alimentos altos en carbohidratos y azúcares, presentando, por tanto, mayor riesgo de desarrollar obesidad (Mooreville et al., 2014). En la misma línea, un estudio realizado con 1660 participantes de población general, en el cual se comparó a pacientes depresivos y no depresivos, se encontró una diferencia entre ellos, tal que participantes depresivos tuvieron una dieta de peor calidad que pacientes no depresivos, controlando por factores sociodemográficos, de estilo de vida y otras comorbilidades psiquiátricas (Rahe et al., 2015).

En la misma dirección, la ansiedad es otro factor psicológico que ha sido identificado como asociada a ingesta de alimentos de alto contenido calórico (Aoun et al., 2019), lo que podría explicarse, por una parte, por el efecto que produce la ansiedad como un estado emocional negativo en la motivación de una conducta alimentaria caracterizada por sobrealimentación (Silva, 2008) o, por otra parte, en cómo el acto de comer supone una reducción de la ansiedad (Dias-Encinas \& Enríquez-Sandoval, 2007). En un reciente estudio, se ha demostrado que personas que puntúan alto en ansiedad, comparadas con quienes puntúan bajo, presentan una mala calidad de la dieta (Gibson-Smith et al., 2018).

En relación con estos antecedentes presentados, se hipotetizó que un factor latente de afectividad negativa, compuesto por estrés psicológico, síntomas depresivos y ansiedad, se asocia directamente con la calidad de la dieta (ver Figura 1, relación C). 


\section{Calidad de la Dieta y Obesidad}

La calidad de la dieta es una de las variables que se ha vinculado con la obesidad (Hall, 2018; Meza \& Moral, 2013). En efecto, se ha reportado que la disponibilidad de alimentos baratos y densos en calorías ha contribuido significativamente al aumento de esta enfermedad (Swinburn et al., 2011). Asimismo, se ha demostrado que adultos norteamericanos que presentan una mayor ingesta de alimentos no saludables, tales como papas fritas, bebidas azucaradas y carnes procesadas, y un menor consumo de frutas y verduras, tienen un mayor peso corporal (Mozaffarian et al., 2011). Similarmente, las prácticas alimentarias de la población chilena se caracterizan por bajo consumo de frutas, verduras, leguminosas, pescados y lácteos y un alto consumo de alimentos industrializados, los que contribuyen a un aporte excesivo de calorías, grasas, azúcares y sal, aumentando con esto las probabilidades de padecer sobrepeso u obesidad (Crovetto \& Uauy, 2010). Consecuentemente, se hipotetizó que la calidad de la dieta tiene una relación directa con la obesidad (ver Figura 1, relación D).

Considerando los antecedentes teóricos y empíricos antes descritos, el presente estudio tuvo como objetivo determinar si existe relación entre la discriminación percibida y la obesidad (operacionalizada como el perímetro de la cintura) en una muestra de adultos chilenos y si esta relación está mediada secuencialmente por la afectividad negativa (definida como estrés psicológico, ansiedad y sintomatología depresiva) y la calidad de la dieta.

\section{Método}

\section{Participantes}

Los participantes de este estudio corresponden a una muestra no probabilística por conveniencia de 420 funcionarios del estamento académico y no académico de una universidad regional estatal del sur de Chile, quienes voluntariamente participaron del proyecto FONDECYT de Iniciación $\mathrm{N}^{\mathrm{o}}$ 11140454. Para efectos de este estudio se consideraron los datos obtenidos en el primer año de ejecución del proyecto. El promedio de edad de los participantes fue de 44 años $(D E=8,79), 248$ participantes eran mujeres y el promedio de índice de masa corporal fue de $27,8(D E=4)$.

Los criterios de exclusión fueron no tener un diagnóstico previo de enfermedad cardiovascular, cáncer, depresión severa o enfermedad inflamatoria, dada su conocida asociación con obesidad y síndrome metabólico.

\section{Instrumentos}

\section{Escala de Discriminación Diaria (Everyday Discrimination Scale, EDS; Williams et al., 1997)}

Es una escala compuesta de nueve ítems que indagan sobre la frecuencia de experiencias de discriminación en la vida cotidiana. Algunos reactivos son: ¿Ha recibido un peor servicio que otras personas? ¿La gente lo ha insultado o puesto sobrenombres? Las opciones de respuesta tienen una escala de frecuencia que oscila entre 0 y 4 puntos, en donde $0=$ nunca, $1=$ casi nunca, $2=$ algunas veces, $3=$ casi siempre y $4=$ siempre. Un mayor puntaje denota un mayor nivel de percepción de discriminación. En este estudio la consistencia interna de la escala, estimada por medio del coeficiente alfa de Cronbach, fue de 0,88. Esta escala no cuenta con estudios de validación en Chile; sin embargo, ha sido utilizada en estudios de similares características, mostrando buenos ajustes psicométricos (Caqueo-Urízar et al., 2019).

\section{Afectividad Negativa}

Escala de Estrés Percibido (Perceived Stress Scale PSS-10; Cohen et al., 1983). Es una escala abreviada de 10 ítems que pregunta a los participantes cuán frecuentemente han experimentado estrés en el último mes. Algunos ejemplos de reactivos son: ¿Cuántas veces se ha molestado por algo que sucedió inesperadamente? ¿Cuántas veces se ha enojado por cosas que estaban fuera de su control? Las opciones de respuesta tienen una escala de frecuencia que varía entre 0 y 4 , en donde $0=$ nunca, $1=$ casi nunca, $2=$ algunas veces, $3=$ casi siempre y $4=$ siempre. Un mayor puntaje denota un mayor nivel de estrés percibido. En este estudio se estimó una consistencia interna, con el coeficiente alfa de Cronbach, de 0,90. Esta escala ha sido previamente validada en población de trabajadores chilenos (Calderón Carvajal, Gómez, López, Otárola \& Briceño, 2017). 
Inventario de Ansiedad Rasgo-Estado (State-Trait Anxiety Inventory, STAI; Spielberger et al., 1970). La STAI está conformada por dos subescalas, ansiedad como estado y ansiedad como rasgo, por lo que frecuentemente ha sido utilizada para medir ambos constructos. Contiene 20 afirmaciones relacionadas con la ansiedad, en donde las personas deben responder con qué frecuencia han experimentado esas situaciones. Algunos ejemplos de ítems son: Tengo un temperamento fuerte, Cuando me enojo digo cosas desagradables. Las opciones de respuesta tienen la siguiente escala de frecuencia: $0=$ nunca, 1 = casi nunca, $2=$ algunas veces, $3=$ casi siempre y $4=$ siempre. En este estudio se utilizaron los 10 ítems de la subescala de ansiedad estado, cuyo mayor puntaje denota un mayor nivel de ansiedad y cuya consistencia interna, estimada con el coeficiente alfa de Cronbach, fue de 0,86. La escala STAI ha sido previamente validada en Chile con estudiantes universitarios (Castillo Pimienta et al., 2016) y en población general 2007), mostrando adecuados coeficientes de confiabilidad.

Escala Revisada de Depresión del Centro de Estudios Epidemiológicos (The Center for Epidemiologic Studies Depression Scale Revised, CES-D; Radloff, 1977). Consiste en una escala que mide a través de 20 afirmaciones con qué frecuencia las personas han experimentado en la última semana una serie de situaciones que dan cuenta de sintomatología depresiva. Algunos ejemplos de ítems son: Me sentí triste a pesar de tener el apoyo de mi familia y amigos o Aunque dormía, al otro día me levantaba cansado. Las opciones de respuesta tienen una escala de frecuencia que varía entre 1 y 4 en donde $1=$ raramente o ninguna vez $($ menos de un día), $2=$ pocas veces $(1-2$ días), $3=$ algunas veces $(3-4$ días $)$ y 4 = la mayoría de las veces (5 - 7 días). Un mayor puntaje denota un mayor nivel de depresión. En este estudio la consistencia interna de la escala, estimada con el coeficiente alfa de Cronbach, fue de 0,92. Las propiedades psicométricas de esta escala han sido previamente estimadas en población juvenil (Gempp Fuentealba, Avendaño Bravo \& Muñoz Urrutia, 2004) y en una muestra normativa y clínica (Gempp Fuentealba \& Thieme, 2010) chilena.

\section{Calidad de la Dieta}

La frecuencia de alimentos ingeridos en los últimos siete días fue autorreportada por medio de un set de preguntas seleccionadas de la Encuesta Nacional de Consumo Alimentario 2010-2011 (Departamento de Nutrición \& Centro de Microdatos, 2011). Para este estudio se consideraron los ítems que midieron consumo de frutas, verduras y comida chatarra, con una frecuencia de respuesta entre 0 (ningún día) a 7 (todos los días), creándose un indicador de mala calidad de la dieta con aquellos tres ítems que midieron un bajo consumo de frutas y verduras y alta ingesta de comida chatarra (Esmaillzadeh et al., 2006; Esposito et al., 2007; Kastorini et al., 2011). Por lo tanto, un mayor puntaje en este indicador corresponde a una peor calidad de la dieta.

\section{Perimetro de Cintura}

El perímetro de cintura fue utilizado para operacionalizar la variable obesidad y fue medido con una cinta métrica modelo Seca 201, aparato de medición que cumple con las exigencias básicas de la directriz 93/42/CEE sobre productos médicos.

\section{Variables Sociodemográficas}

Potenciales covariables fueron evaluadas. Mediante autorreporte, los participantes indicaron su edad, sexo, condiciones médicas y medicación prescrita, en caso de existir.

\section{Covariables}

Tanto el nivel socioeconómico (NSE) como el sexo se incluyeron como covariables. Era necesario controlar estadísticamente estas variables, pues es posible que parte de la varianza de la variable dependiente (perímetro de cintura) sea explicada por ellas. De esta forma, se esperaba que todas las relaciones estimadas en el análisis, en caso de ser significativas, lo sean independiente del NSE y el sexo de los participantes, para asegurar que los resultados puedan ser generalizados a hombres y mujeres con distintos NSE.

El NSE de los participantes se midió utilizando tres instrumentos que dieron cuenta del ingreso mensual (¿cuál es su ingreso mensual líquido individual?), máximo nivel de estudios alcanzado (¿cuál es su 
nivel de estudios más alto alcanzado?, donde el nivel más bajo corresponde a educación básica completa y el más alto, grado académico de doctor) y la percepción subjetiva de posición social (métrica de 6 peldaños, donde $1=$ bajo, $2=$ medio bajo, $3=$ medio, $4=$ medio alto, $5=$ alto y $6=$ muy alto $)($ Adler et al., 2000).

\section{Procedimiento}

El procedimiento que a continuación se describe corre sponde al Proyecto FONDECYT de Iniciación $\mathrm{N}^{0}$ 11140454, del cual fueron extraído los datos para este estudio. Para efectos de este estudio, solo se utilizaron las variables de interés antes descritas.

Todo el procedimiento fue evaluado y aprobado por el respectivo Comité Ético Científico de una universidad regional estatal del sur de Chile. Los participantes asistieron con una ayuna de al menos ocho horas al Laboratorio de Estrés y Salud, en donde ayudantes de investigación explicaron los objetivos del estudio y entregaron el documento de consentimiento informado, el cual fue firmado por cada participante. Posteriormente, técnicos en enfermería practicaron a cada participante un examen de sangre venosa para determinar su perfil bioquímico, mediante el uso de un dispositivo BD Vacutainer ${ }^{\circledR}$ UltraTouch ${ }^{\mathrm{TM}}$ Push Button Blood Collection Set. Además, estudiantes de posgrado debidamente capacitadas tomaron medidas antropométricas de peso, talla y perímetro de cintura y otras tres medidas de presión arterial. Finalmente, cada participante respondió las escalas para medir variables psicológicas de interés en formato físico. Una vez terminado el procedimiento, cada participante fue retribuido económicamente con ocho mil pesos ( 13 dólares americanos). Los resultados de medidas antropométricas, presión arterial y perfil bioquímico fueron devueltos a cada participante.

\section{Plan de Análisis}

Para determinar efectos directos e indirectos se realizaron análisis de ecuaciones estructurales. Este análisis permite modelar la relación entre factores latentes y variables observadas y estimar efectos directos y mediados (Ortiz \& Fernández-Pera, 2018).

Previo al análisis de ecuaciones estructurales, se crearon dos factores latentes, uno para afectividad negativa y otro para calidad de la dieta. En el caso del primer factor latente, sus indicadores fueron estrés psicológico, sintomatología depresiva y ansiedad, y para el segundo factor se consideró como indicadores el consumo de frutas, consumo de vegetales e ingesta de comida chatarra. En ambos casos, el análisis factorial confirmatorio permite identificar una estructura factorial, con cargas factoriales significativas $(p<0,001) \mathrm{y}$ adecuadas (rango 0,31 a 0,84).

Posteriormente, se realizó el análisis de ecuaciones estructurales con el método de máximo verosimilitud e incorporando la corrección de Satorra-Bentler, dado el incumplimiento del supuesto de normalidad multivariada (test multivariado de Mardia $p<0,001$ ).

Para el ajuste global del modelo, se utilizaron indicadores de bondad de ajuste convencionales: $\chi^{2}$ no significativo, el cociente entre $\chi^{2}$ y sus grados de libertad $\left(\chi^{2} / g l\right)<2$, el índice comparativo (CFI) igual o superior a 0,95, el índice de Tucker Lewis (TLI) igual o superior a 0,90, la raíz del residuo cuadrático medio estandarizado (SRMR) igual o inferior a 0,08 y la raíz del error cuadrático medio de aproximación (RMSEA) igual o inferior a 0,06 .

En todas las relaciones analizadas se controló por las covariables NSE y sexo. Se reportan los coeficientes estandarizados.

Los análisis fueron realizados con el software STATA 14.2 y consideraron un alfa nominal de 0,05.

\section{Resultados}

El 56\% de los sujetos se posiciona en la categoría de NSE medio. Treinta por ciento de los participantes posee educación media incompleta/completa, 25\%, una carrera técnica incompleta/completa, 16\%, una carrera universitaria incompleta/completa y $29 \%$, estudios de posgrado. El $12 \%$ de los participantes reportó tener un ingreso económico mensual inferior a 250 mil pesos chilenos ( 420 dólares americanos), 44\%, un sueldo entre 251 mil y 500 mil pesos ( 421 a 840 dólares americanos), 31\%, un ingreso entre 501 mil y 1 millón 500 mil pesos ( 842 a 2521 dólares americanos) y 13\%, un ingreso por sobre 1 millón 500 mil pesos (más de 2521 dólares americanos). 
En relación a las hipótesis del estudio, se observa un efecto directo de la percepción de discriminación en la afectividad negativa, tal que a mayor discriminación percibida mayor es la afectividad negativa $(B=$ 0,470, $p=0,001)$. A su vez, este factor de afectividad negativa se asoció directamente con el factor de calidad de la dieta $(B=0,220, p=0,001)$, lo cual implica un menor consumo de frutas y vegetales y mayor consumo de comida chatarra. Finalmente, el factor de calidad de la dieta se asoció con mayor perímetro de cintura $(B=0,151, p=0,001)$. No hubo efecto directo de la percepción de discriminación en el perímetro de cintura $(B=0,073, p=0,122)$ (ver Figura 2 ) y fue totalmente mediada por la secuencia de mediación propuesta. Específicamente, y con respecto a los efectos indirectos, se observa que la percepción de discriminación se asocia con la calidad de la dieta, vía afectividad negativa ( $B_{\text {indirecto }}=0,103, p=0,048$ ). Asimismo, existe un efecto indirecto de la afectividad negativa en el perímetro de cintura, por medio de la calidad de la dieta $\left(B_{\text {indirecto }}=0,033, p=0,044\right)$. Finalmente, hubo un efecto indirecto de la percepción de discriminación en el perímetro de cintura vía afectividad negativa y calidad de la dieta ( $B_{\text {indirecto }}=0,015, p=$ 0,049). El efecto total de la discriminación en el perímetro de cintura fue $0,99(p=0,030)$.

Los indicadores de bondad de ajuste para este modelo fueron los siguientes: $\chi^{2}(17, n=420)=30,27$, $p=0,020, \chi^{2} / g l=1,78, \mathrm{CFI}=0,980, \mathrm{TLI}=0,970, \mathrm{RMSEA}=0,043$, SRMR $=0,036$. El coeficiente de determinación fue 0,231. Estos indicadores dan cuenta de un buen ajuste global del modelo a los datos.

\section{Discusión}

Este estudio tuvo por finalidad determinar si la discriminación general percibida se relaciona directamente con obesidad y si esta relación está mediada por la afectividad negativa y por las características de la dieta en adultos chilenos. Respecto a estos objetivos, los resultados dan cuenta que, si bien no existe un efecto directo de la discriminación en el perímetro de cintura (utilizado en este estudio como un proxy de obesidad), existe un efecto indirecto mediado por la afectividad negativa y la calidad de la dieta y que, pese a tener una magnitud pequeña, permite identificar una secuencia de mediación que vincula variables psicológicas con desenlaces en salud, tales como la obesidad.

\section{Figura 2}

Modelo de Relación entre Percepción de Discriminación y Perímetro de Cintura

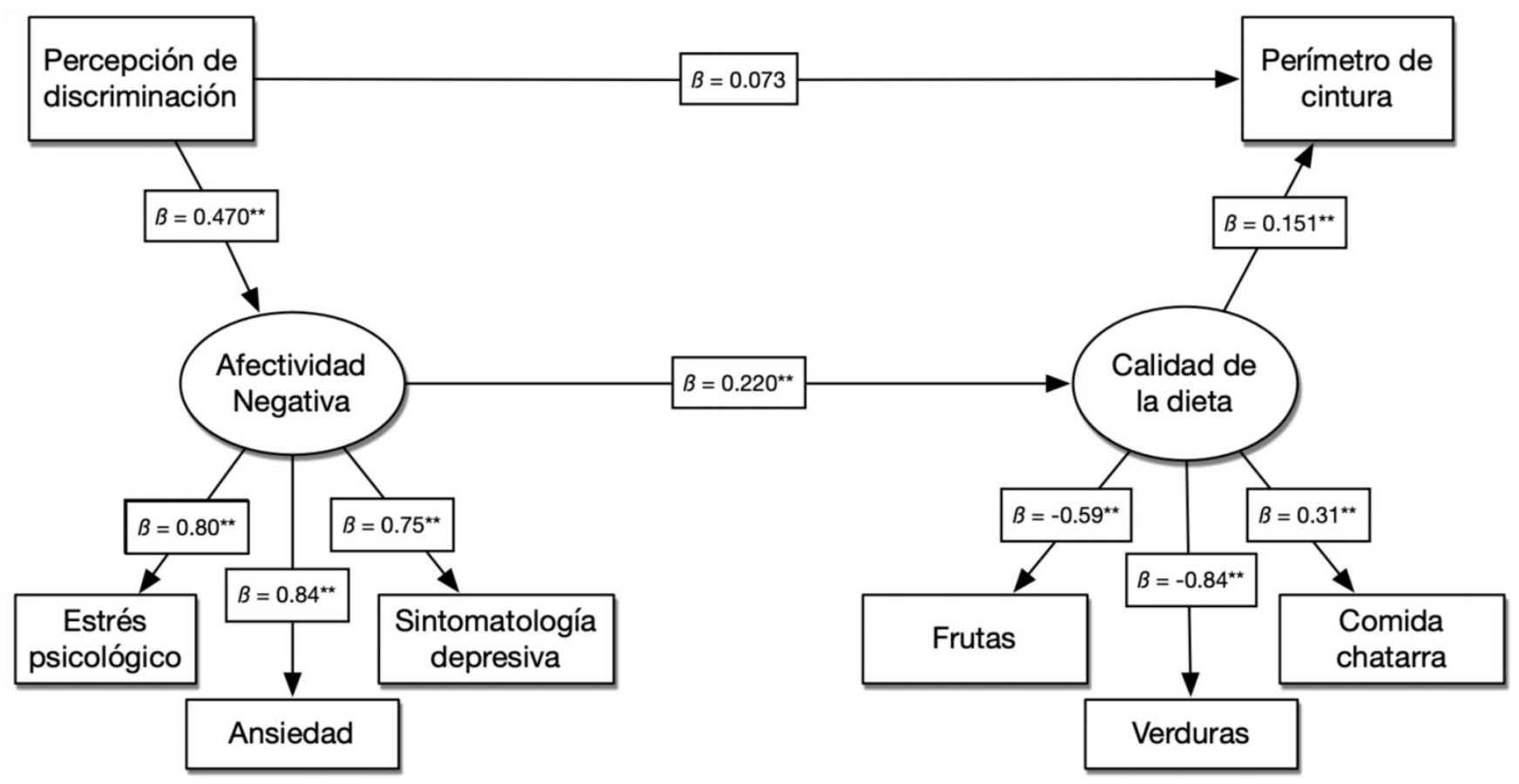

Nota. ${ }^{* *} p=0,001$. 
En este estudio no se identificó un efecto directo de la percepción de discriminación en el perímetro de cintura, sino un efecto completamente mediado por la afectividad negativa y la calidad de la dieta. Este resultado está en línea con estudios previos que señalan que para que la percepción de discriminación se asocie con obesidad debe ocurrir una serie de procesos tanto fisiológicos como conductuales. De esta forma, la percepción de discriminación desencadenará la desregulación de sistemas fisiológicos, tales como la activación del eje hipotalámico hipofisario adrenal, y se asociará con conductas no saludables (Adam \& Epel, 2007). En forma similar, estudios previos indican que la discriminación percibida se asocia con la obesidad a través de procesos psicológicos y conductuales (Johnson et al., 2012; Shelton et al., 2009), tales como la afectividad negativa y la preferencia por alimentos no saludables. En efecto, en este estudio la percepción de discriminación se asoció con afectividad negativa, caracterizada por altos niveles de estrés psicológico, ansiedad y síntomas depresivos, lo cual destaca las consecuencias negativas que la discriminación percibida tiene en la salud mental de personas objeto de tal discriminación y confirma hallazgos previos en esta línea (Carter et al., 2016; Cheng et al., 2015; Tomiyama et al., 2015). El presente estudio también demuestra que la afectividad negativa se asocia con una peor calidad de la dieta, lo que se relaciona con hallazgos previos que evidencian la existencia de una relación entre estrés psicológico (Isasi et al., 2015), depresión y ansiedad (Gibson-Smith et al., 2018) con una mala calidad de la dieta.

El resultado antes discutido es relevante, pues permite identificar un mecanismo poco estudiado, que asocia una variable psicológica con un desenlace negativo en salud, pero más aún, revela las consecuencias psicológicas y conductuales que esta experiencia tiene. La percepción de discriminación tiene características de un evento altamente estresante, tales como la evaluación social, comúnmente basada en características físicas visibles y, además, la incontrolabilidad. Estas características de la discriminación tienen consecuencias severas en la salud de las personas, más que otro tipo de estresores psicológicos (Ortiz et al., 2014).

Los resultados de este estudio pueden ser de utilidad al momento de pensar estrategias de intervención para personas que son víctimas de discriminación y prejuicio y, en especial, aquellas que son discriminadas por su sobrepeso u obesidad. Producto que las principales características de la obesidad son visibles y que las personas obesas son frecuentemente discriminadas y estigmatizadas por su peso (Tomiyama, 2014), es altamente probable que ellas experimenten afectividad negativa, lo que puede generar comportamientos no saludables, tales como ingerir una dieta de mala calidad (Richardson et al., 2015; Whitaker et al., 2014). De esta forma, intervenciones orientadas a la pérdida de peso debieran integrar estrategias a nivel individual y social. Dentro de las primeras, a la práctica regular de actividad física, se deben sumar entrenamientos en mecanismos activos de afrontamiento al estrés psicológico, tratamiento de sintomatología depresiva y/o ansiosa y la educación en pautas de alimentación saludable y, dentro de las segundas, educación a la población general sobre las negativas consecuencias que tiene la discriminación por peso.

El presente estudio tuvo fortalezas y debilidades. Una de sus fortalezas es el tamaño muestral, el cual permitió contar con una adecuada potencia estadística para detectar efectos estadísticamente significativos. Asimismo, el uso de ecuaciones estructurales facilitó la comprobación de las hipótesis y la estimación simultánea de efectos directos e indirectos en el modelo. En relación a las debilidades, una de ellas tiene relación con que la medida de percepción de discriminación utilizada en este estudio: es general y no permitió distinguir entre diferentes tipos o causas de discriminación. De haber sido posible, se podrían haber comparado modelos con diferentes atribuciones de discriminación y estimar efectos diferenciales en base a diferentes causas o motivos de discriminación. Pese a esto, y con independencia de la causa a la cual se atribuya ser discriminado, los resultados obtenidos en este estudio permiten afirmar que una mayor frecuencia de discriminación se asocia con mayor afectividad negativa, caracterizada por estrés psicológico, ansiedad y síntomas depresivos.

El diseño transversal del estudio podría ser considerado como una limitación que podría atentar contra la posibilidad de establecer relaciones de causalidad y realizar el análisis de mediación. En este sentido, se asume que las relaciones postuladas en este estudio están fuertemente orientadas por la teoría y por resultados de estudios previos, permitiendo con esto establecer relaciones de causalidad teórica. De esta forma, es más probable que personas que perciben discriminación, por ejemplo, por su peso, apariencia física o NSE, experimenten afectividad negativa caracterizada por estrés psicológico, ansiedad y síntomas depresivos y no al contrario (Gómez-Pérez et al., 2017). Por otra parte, y de acuerdo a los postulados de Hayes (2013), es factible realizar análisis de mediación en diseños transversales, siempre y cuando las relaciones postuladas tengan un fuerte sustento teórico y empírico, situación que ocurre en este estudio. 
Finalmente, y en relación a estas limitaciones, futuros estudios deberían considerar la ejecución de un estudio longitudinal de panel que permita evaluar el rol que tienen variables psicológicas, tales como la percepción de discriminación, en los cambios y/o trayectorias que asumen en el tiempo marcadores objetivos de obesidad. Además de lo anterior, es necesario identificar la atribución que los participantes hacen de tal experiencia, pues podría ocurrir que las personas sean objeto de discriminación por más de una causa (por ejemplo, peso y sexo), produciendo una doble discriminación. La correcta identificación de las causas que generan discriminación permitiría tener una comprensión más compleja del fenómeno, así como también detectar posibles efectos de interacción entre variables.

\section{Referencias}

Adam, T. C. \& Epel, E. S. (2007). Stress, eating and the reward system. Physiology \& Behavior, 91(4), 449-458. https://doi.org/10.1016/j.physbeh.2007.04.011

Adler, N. E., Epel, E. S., Castellazzo, G. \& Ickovics, J. R. (2000). Relationship of subjective and objective social status with psychological and physiological functioning: Preliminary data in healthy white women. Health Psychology, 19(6), 586-592. https://doi.org/10.1037//0278-6133.19.6.586

Aoun, C., Nassar, L., Soumi, S., El Osta, N., Papazian, T. \& Khabbaz, L. R. (2019). The cognitive, behavioral, and emotional aspects of eating habits and association with impulsivity, chronotype, anxiety, and depression: A cross-sectional study. Frontiers in Behavioral Neuroscience, 13(13), Article 204. https://doi.org/10.3389/fnbeh.2019.00204

Araiza, A. M. \& Lobel, M. (2018). Stress and eating: Definitions, findings, explanations, and implications. Social and Personality Psychology Compass, 12(4), Article e12378. https://doi.org/10.1111/spc3.12378

Calderón Carvajal, C., Gómez, N., López, F., Otárola, N. \& Briceño, M. (2017). Estructura factorial de la Escala de Estrés Percibido (PSS) en una muestra de trabajadores chilenos. Salud \& Sociedad, 8(3), 218-226. https://doi.org/10.22199/S07187475.2017.0003.00002

Caqueo-Urízar, A., Flores, J., Irarrázaval, M., Loo, N., Páez, J. \& Sepúlveda, G. (2019). Discriminación percibida en escolares migrantes en el norte de Chile. Terapia Psicológica, 37(2), 97-103. https://doi.org/10.4067/S0718-48082019000200097

Carter, S. E., Walker, R. L., Cutrona, C. E., Simons, R. L. \& Beach, S. R. H. (2016). Anxiety mediates perceived discrimination and health in African-American women. American Journal of Health Behavior, 40(6), 697-704. https://doi.org/10.5993/AJHB.40.6.2

Castillo Pimienta, C., Chacón de La Cruz, T. \& Díaz-Véliz, G. (2016). Ansiedad y fuentes de estrés académico en estudiantes de carreras de la salud. Investigación en Educación Médica, 5(20), 230-237. https://doi.org/10.1016/j.riem.2016.03.001

Cheng, E. R., Cohen, A. \& Goodman, E. (2015). The role of perceived discrimination during childhood and adolescence in understanding racial and socioeconomic influences on depression in young adulthood. Journal of Pediatrics, 166(2), 370-377. https://doi.org/10.1016/j.jpeds.2014.10.010

Cohen, S., Kamarck, T. \& Mermelstein, R. (1983). A global measure of perceived stress. Journal of Health and Social Behavior, 24(4), 385-396. https://doi.org/10.2307/2136404

Crovetto, M. \& Uauy, R. (2010). Cambios en el consumo aparente de nutrientes en el Gran Santiago 1988-1997 en hogares según ingreso y su probable relación con patrón de enfermedades crónicas no transmisibles. Revista Médica de Chile, 138(9), $1091-1108$. https://doi.org/10.4067/S0034-98872010000900003

Departamento de Nutrición \& Centro de Microdatos. (2011). Anexos Encuesta Nacional de Consumo Alimentario. Universidad de Chile, Facultad de Medicina, Escuela de Salud Pública, Escuela de Nutrición y Facultad de Econocomía y Negocios. https://www.minsal.cl/sites/default/files/ANEXOS_ENCA.pdf

Dias-Encinas, D. R. \& Enríquez-Sandoval, D. R. (2007). Obesidad infantil, ansiedad y familia. Boletín Clínico Hospital Infantil del Estado de Sonora, 24(1), 22-26. https://www.medigraphic.com/pdfs/bolclinhosinfson/bis-2007/bis071e.pdf

Esmaillzadeh, A., Kimiagar, M., Mehrabi, Y., Azadbakht, L., Hu, F. B. \& Willett, W. C. (2006). Fruit and vegetable intakes, C-reactive protein, and the metabolic syndrome. The American Journal of Clinical Nutrition, 84(6), 1489-1497. https://doi.org/10.1093/ajen/84.6.1489

Esposito, K., Ceriello, A. \& Giugliano, D. (2007). Diet and the metabolic syndrome. Metabolic Syndrome and Related Disorders, 5(4), 291-296. https://doi.org/10.1089/met.2007.0018

Finch, L. E. \& Tomiyama, A. J. (2015). Comfort eating, psychological stress, and depressive symptoms in young adult women. Appetite, 95, 239-244. https://doi.org/10.1016/j.appet.2015.07.017

Gempp Fuentealba, R., Avendaño Bravo, C. \& Muñoz Urrutia, C. (2004). Normas y punto de corte para la Escala de Depresión del Centro para Estudios Epidemiológicos (CES-D) en población juvenil chilena. Terapia Psicológica, 22(2), $145-156$. https://www.redalyc.org/articulo.oa?id=78522205

Gempp Fuentealba, R. \& Thieme, C. (2010). Efecto de diferentes métodos de puntuación sobre la fiabilidad, validez y puntos de corte de la Escala de Depresión del Centro para Estudios Epidemiológicos (CES-D). Terapia Psicológica, $28(1), \quad 5-12$. https://doi.org/10.4067/s0718-48082010000100001

Gibson-Smith, D., Bot, M., Brouwer, I. A., Visser, M. \& Penninx, B. W. J. H. (2018). Diet quality in persons with and without depressive and anxiety disorders. Journal of Psychiatric Research, 106, 1-7. https://doi.org/10.1016/j.jpsychires.2018.09.006

Gómez-Pérez, D., Ortiz, M. S. \& Saiz, J. L. (2017). Estigma de obesidad, su impacto en las víctimas y en los equipos de salud: una revisión de la literatura. Revista Medica de Chile, 145(9), 1160-1164. https://doi.org/10.4067/s0034-98872017000901160

Hall, K. D. (2018). Did the food environment cause the obesity epidemic? Obesity(1), 26, 11-13. https://doi.org/10.1002/oby.22073

Hatzenbuehler, M. L., Keyes, K. M. \& Hasin, D. S. (2009). Associations between perceived weight discrimination and the prevalence of psychiatric disorders in the general population. Obesity, 17(11), 2033-2039. https://doi.org/10.1038/oby.2009.131

Hayes, A. F. (2013). Introduction to mediation, moderation, and conditional process analysis: A regression-based approach. Guilford Press. https://doi.org/978-1-60918-230-4 
Hunte, H. E. R. (2011). Association between perceived interpersonal everyday discrimination and waist circumference over a 9-year period in the midlife development in the United States cohort study. American Journal of Epidemiology, 173(11), 1232-1239. https://doi.org/10.1093/aje/kwq463

Isasi, C. R., Parrinello, C. M., Jung, M. M., Carnethon, M. R., Birnbaum-Weitzman, O., Espinoza, R. A., Penedo, F. J., Perreira, K. M., Schneiderman, N., Sotres-Alvarez, D., Van Horn, L. \& Gallo, L. C. (2015). Psychosocial stress is associated with obesity and diet quality in Hispanic/Latino adults. Annals of Epidemiology, 25(2), 84-89. https://doi.org/10.1016/j.annepidem.2014.11.002

Jakobsen, G. S., Småstuen, M. C., Sandbu, R., Nordstrand, N., Hofsø, D., Lindberg, M., Hertel, J. K. \& Hjelmesæth, J. (2018). Association of bariatric surgery vs medical obesity treatment with long-term medical complications and obesity-related comorbidities. JAMA: The Journal of the American Medical Association, 319(3), 291-301. https://doi.org/10.1001/jama.2017.21055

Johnson, P., Risica, P. M., Gans, K. M., Kirtania, U. \& Kumanyika, S. K. (2012). Association of perceived racial discrimination with eating behaviors and obesity among participants of the SisterTalk study. Journal of National Black Nurses' Association, 23(1), 34-40. https://www.ncbi.nlm.nih.gov/pmc/articles/PMC3773811/?report=abstract/pdf/nihms481954.pdf

Joseph, P. V., Davidson, H. R., Boulineaux, C. M., Fourie, N. H., Franks, A. T., Abey, S. K. \& Henderson, W. A. (2018). Eating behavior, stress, and adiposity: Discordance between perception and physiology. Biological Research for Nursing, 20(5), 531-540. https://doi.org/10.1177/1099800418779460

Kastorini, C. -M., Milionis, H. J., Esposito, K., Giugliano, D., Goudevenos, J. A. \& Panagiotakos, D. B. (2011). The effect of Mediterranean diet on metabolic syndrome and its components: A meta-analysis of 50 studies and 534,906 individuals. Journal of the American College of Cardiology, 57(1), 1299-1313. https://doi.org/10.1016/j.jacc.2010.09.073

Lewis, T. T., Kravitz, H. M., Janssen, I. \& Powell, L. H. (2011). Self-reported experiences of discrimination and visceral fat in middleaged African-American and Caucasian women. American Journal of Epidemiology, 173(11), 1223-1231. https://doi.org/10.1093/aje/kwq466

Meza, C. \& Moral, J. (2013). Modelos recursivos de índice de masa corporal con variables sociodemográficas, funciones del yo y alteración alimentaria en mujeres mexicanas. Psykhe, 22(1), 55-68. https://doi.org/10.7764/psykhe.22.1.593

Mooreville, M., Shomaker, L. B., Reina, S. A., Hannallah, L. M., Cohen, L. A., Courville, A. B., Kozlosky, M., Brady, S. M., Condarco, T., Yanovski, S. Z., Tanofsky-Kraff, M. \& Yanovski, J. A. (2014). Depressive symptoms and observed eating in youth. Appetite, 75, 141-149. https://doi.org/10.1016/j.appet.2013.12.024

Mozaffarian, D., Hoa, T., Rimm, E. B., Willett, W. C. \& Hu, F. B. (2011). Changes in diet and lifestyle and long-term weight gain in women and men. The New England Journal of Medicine, 364(25), 2392-2404. https://doi.org/10.1056/NEJMoa1014296

Organización de las Naciones Unidas para la Alimentación y la Agricultura \& Organización Panamericana de la Salud. (2017). 2016 América Latina y el Caribe: panorama de la seguridad alimentaria y nutricional. Sistemas alimentarios sostenibles para poner fin al hambre y la malnutrición. http://www.fao.org/3/a-i6747s.pdf

Ortiz, M. S. \& Fernández-Pera, M. (2018). Modelo de ecuaciones estructurales: una guía para ciencias médicas y ciencias de la salud. Terapia Psicológica, 36(1), 51-58. https://doi.org/10.4067/s0718-48082017000300047

Ortiz, M. S., Willey, J. F. \& Chiang, J. J. (2014). How stress gets under the skin o cómo el estrés psicológico se introduce bajo la piel. Revista Medica de Chile, 142(6), 767-774. https://doi.org/10.4067/S0034-98872014000600011

Paradies, Y. (2006). A systematic review of empirical research on self-reported racism and health. International Journal of Epidemiology, 35(4), 888-901. https://doi.org/10.1093/ije/dyl056

Pascoe, E. A. \& Richman, L. (2009). Perceived discrimination and health: A meta-analytic review. Psychological Bulletin, 135(4), 531554. https://doi.org/10.1037/a0016059

Radloff, L. S. (1977). The CES-D Scale: A self-report depression scale for research in the general population. Applied Psychological Measurement, 1(3), 385-401. https://doi.org/10.1177/014662167700100306

Rahe, C., Baune, B. T., Unrath, M., Arolt, V., Wellmann, J., Wersching, H. \& Berger, K. (2015). Associations between depression subtypes, depression severity and diet quality: Cross-sectional findings from the BiDirect Study. BMC Psychiatry, 15, Article 38. https://doi.org/10.1186/s12888-015-0426-9

Richardson, A. S., Arsenault, J. E., Cates, S. C. \& Muth, M. K. (2015). Perceived stress, unhealthy eating behaviors, and severe obesity in low-income women. Nutrition Journal, 14, Article 122. https://doi.org/10.1186/s12937-015-0110-4

Schmitt, M. T., Branscombe, N. R., Postmes, T. \& Garcia, A. (2014). The consequences of perceived discrimination for psychological well-being: A meta-analytic review. Psychological Bulletin, 140(4), 921-948. https://doi.org/10.1037/a0035754

Shelton, R. C., Puleo, E., Bennett, G. G., McNeill, L. H., Sorensen, G. \& Emmons, K. M. (2009). The association between racial and gender discrimination and body mass index among residents living in lower-income housing. Ethnicity \& Disease, 19(3), $251-257$. https://www.ncbi.nlm.nih.gov/pmc/articles/PMC2855131/pdf/nihms-186497.pdf

Silva, J. R. (2008). Sobrealimentación inducida por la ansiedad, parte II: Un marco de referencia neurocientífico para el desarrollo de técnicas psicoterapéuticas y programas de prevención. Terapia Psicológica, 26(1), 99-115. https://doi.org/10.4067/S0718$\underline{48082008000100009}$

Smith, K. B. \& Smith, M. S. (2016). Obesity statistics. Primary Care: Clinics in Office Practice, 43(1), 121-135. https://doi.org/10.1016/j.pop.2015.10.001

Spielberger, C. D., Gorsuch, R. L. \& Lushene, R. E. (1970). Manual for the State-Trait Anxiety Inventory. Consulting Psychologists Press.

Stepanikova, I., Baker, E. H., Simoni, Z. R., Zhu, A., Rutland, S. B., Sims, M. \& Wilkinson, L. L. (2017). The role of perceived discrimination in obesity among African Americans. American Journal of Preventive Medicine, 52(Supplement 1), S77-S85. https://doi.org/10.1016/j.amepre.2016.07.034

Sutin, A. R. \& Terracciano, A. (2013). Perceived weight discrimination and obesity. PLoS One, 8(7), Article e70048. https://doi.org/10.1371/journal.pone.0070048

Swinburn, B. A., Sacks, G., Hall, K. D., McPherson, K., Finegood, D. T., Moodie, M. L. \& Gortmaker, S. L. (2011). The global obesity pandemic: Shaped by global drivers and local environments. The Lancet, 378(9793), 804-814. https://doi.org/10.1016/S0140$\underline{6736(11) 60813-1}$

Tomiyama, A. J. (2014). Weight stigma is stressful. A review of evidence for the cyclic obesity/weight-based stigma model. Appetite, 82, 8-15. https://doi.org/10.1016/j.appet.2014.06.108 
Tomiyama, A. J., Finch, L. E. \& Cummings, J. R. (2015). Did that brownie do its job? Stress, eating, and the biobehavioral effects of comfort food. En R. A. Scott \& S. M. Kosslyn (Eds.), Emerging trends in the social and behavioral sciences (pp. 1-15). John Wiley \& Sons. https://doi.org/10.1002/9781118900772.etrds0324

Vera-Villarroel, P., Celis-Atenas, K., Córdova-Rubio, N., Buela-Casal, G. \& Spielberger, C. D. (2007). Preliminary analysis and normative data of the State-Trait Anxiety Inventory (STAI) in adolescent and adults of Santiago, Chile. Terapia Psicológica, 25(2), 155-162. https://doi.org/10.4067/s0718-48082007000200006

Whitaker, K. M., Sharpe, P. A., Wilcox, S. \& Hutto, B. E. (2014). Depressive symptoms are associated with dietary intake but not physical activity among overweight and obese women from disadvantaged neighborhoods. Nutrition Research, 34(4), $294-301$. https://doi.org/10.1016/i.nutres.2014.01.007

Williams, D. R., Yu, Y., Jackson, J. S. \& Anderson, N. B. (1997). Racial differences in physical and mental health: Socio-economic status, stress and discrimination. Journal of Health Psychology, 2(3), 335-351. https://doi.org/10.1177/135910539700200305

Wright, S. M. \& Aronne, L. J. (2012). Causes of obesity. Abdominal Radiology, 37(5), 730-732. https://doi.org/10.1007/s00261-012-9862-x

Zeiders, K. H., Doane, L. D. \& Roosa, M. W. (2012). Perceived discrimination and diurnal cortisol: Examining relations among Mexican American adolescents. Hormones and Behavior, 61(4), 541-548. https://doi.org/10.1016/j.yhbeh.2012.01.018

Fecha de recepción: Abril de 2019.

Fecha de aceptación: Abril de 2020. 